\title{
Homologous recombination in human embryonic stem cells using CRISPR/Cas9 nickase and a long DNA donor template
}

\section{Dear Editor,}

Genome editing of human embryonic stem cells (hESCs) is critical for basic biological research and regenerative medicine. However, until a few years ago, gene targeting technologies to disrupt, repair or overexpress genes in hESCs had been very inefficient and thus could not be routinely used. Recent technical breakthrough includes bacterial artificial chromosome based high efficiency gene targeting (Song et al., 2010), as well as the successful engineering of two systems of site-specific nucleases, zinc-finger nucleases (ZFNs) and transcription activator-like effector nucleases (TALENs) (Hockemeyer et al., 2009; Hockemeyer et al., 2011). The two engineered nucleases are composed of programmable and sequence-specific DNA-binding modules, which bring the nucleases to specific genomic site to introduce a DNA double-strand break. However, these two technologies have several limitations, including the timeconsuming and labor-intensive experimental design, and the risk of off-targeting mutations (Gaj et al., 2013). More recently, a new genome-editing technology, denoted the clustered regularly interspaced short palindromic repeats (CRISPR)/CRISPR-associated (Cas) system, has been developed for efficient gene targeting in cells of various species, including zebrafish (Hwang et al., 2013), mouse (Wang et al., 2013), monkey (Niu et al., 2014), and human (Cong et al., 2013; Mali et al., 2013b). In this system, Cas9 nuclease is targeted to a specific genomic site by complexing with a guide RNA, which hybridizes a 20-nucleotide DNA sequence immediately preceding an NGG motif, introducing a double-strand break three nucleotides upstream of the NGG motif (Jinek et al., 2012). Compared to ZFNs and TALENs, CRISPR/Cas9 system offers simple experimental design and very high targeting efficiency (Ding et al., 2013). However, some studies have also raised the concern about the off-target mutation effect of this system (Hsu et al., 2013; Mali et al., 2013a). To reduce the off-target mutations, a D10A mutant nickase version of Cas9 (Cas9n) has been developed to replace wild type Cas 9 and shown to increase the ratio of homology-directed repair (HDR) to nonhomologous end joining (NHEJ) (Cong et al., 2013; Mali et al., 2013b). However, the targeting efficiency of Cas9n is much lower than wild type Cas9, raising the concern that it cannot be applied in homologous recombination (HR) in hESCs.

To test whether Cas9n can induce precise gene targeting via homologous recombination at a gene locus not expressed in hESCs, we designed the experiment to employ Cas9n and a long DNA donor template to introduce a drug resistance gene into a lineage-specific locus. We picked the gene locus of pulmonary surfactant protein C (SFTPC), which is specifically expressed in pulmonary alveolar type II cells (Degryse et al., 1996). In the long DNA donor template, an IRES-PuropA expression cassette was inserted between the stop codon and the polyA signal sequence of SFTPC gene with two homologous arms around $1 \mathrm{~kb}$. sgRNA-1 (\#1 in Fig. 1A), which targeted the site about 170 bps upstream of the junction site of the two homologous arms, was designed according to the UCSC genome browser tracks developed by Zhang lab at MIT. While, sgRNA-2 (\#2 in Fig. 1A), which targeted the junction site, was designed according to $(\mathrm{N})_{20} \mathrm{NGG}$ rule. The Cas9n expression cassette, sgRNAs and donor DNA were electroporated into HUES3 hESCs. After puromycin selection, 37 and 36 clones survived with the transfection of sgRNA-1 and sgRNA-2, respectively. Five clones derived from sgRNA-1 transfection were found to be homologous recombinants at the upstream arm by PCR screening, while no clone derived from sgRNA-2 transfection was homologous recombinant (Fig. 1F). To confirm that the clones with homologous recombination at $5^{\prime}$ homologous arm do not harbor random integration, the genomic DNA was examined by Southern blotting with the upstream, downstream and internal probes, and with two restriction enzymes. As shown in Fig. 1D, two clones were identified to have precise HR at both arms and no random integration. The other three clones were identified to have single-crossover at the upstream homologous arm (data not shown). By transiently expressing Flp, the F5 flanked selection cassette was removed, leading to the knock-in allele (Fig. 1E).

In this study, we demonstrate that Cas9n can induce precise gene targeting via HR in hESCs with a long DNA donor template. The targeting efficiency is around $5 \%(2 / 37)$ in HUES3 cells (Fig.1F). In contrast to the oligonucleotide- 
A

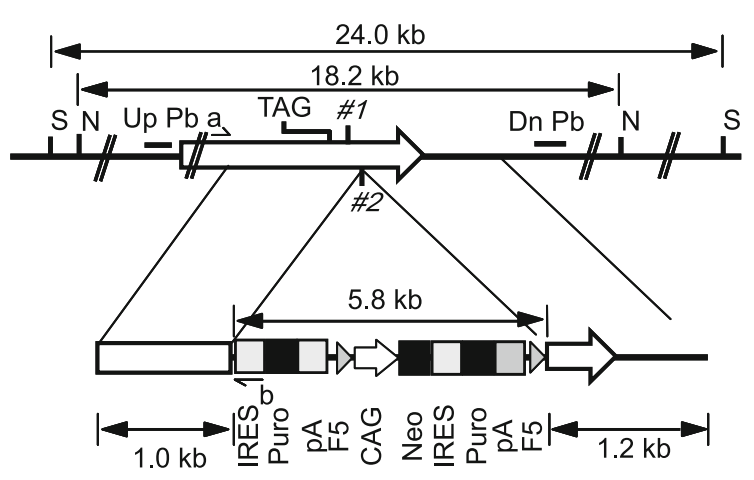

C

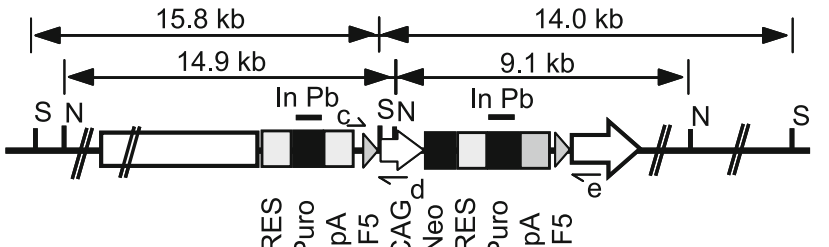

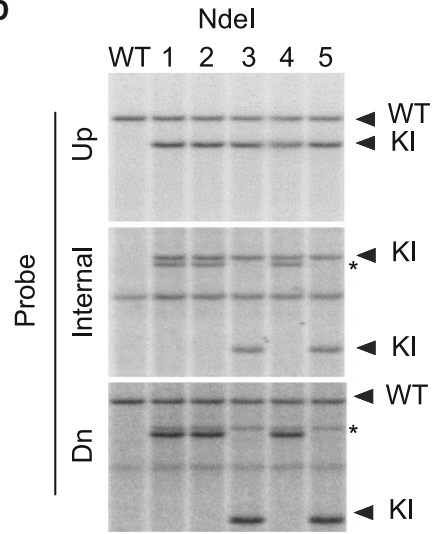



E

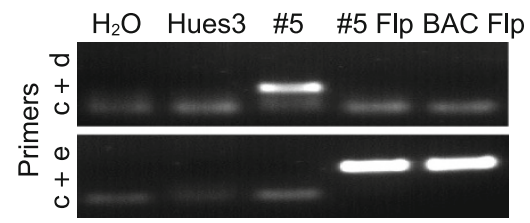

$\mathbf{F}$

\begin{tabular}{|c|c|c|c|c|c|c|c|c|}
\hline \multirow[b]{2}{*}{ Cell line } & \multirow[b]{2}{*}{$\begin{array}{l}\text { Guide } \\
\text { RNA }\end{array}$} & \multirow{2}{*}{$\begin{array}{c}\text { Selection } \\
\text { resistant } \\
\text { clone }\end{array}$} & \multirow{2}{*}{$\begin{array}{l}\text { Positive } \\
\text { clone in } \\
\text { PCR } \\
\text { screen }\end{array}$} & \multicolumn{3}{|c|}{ Positive clone in Southern blotting } & \multirow[b]{2}{*}{$\begin{array}{c}\text { Correct } \\
\text { knockin } \\
\text { clone }\end{array}$} & \multirow[b]{2}{*}{$\begin{array}{l}\text { Targeting } \\
\text { efficiency }\end{array}$} \\
\hline & & & & $\begin{array}{l}\text { Upstream } \\
\text { probe }\end{array}$ & $\begin{array}{l}\text { Downstream } \\
\text { probe }\end{array}$ & $\begin{array}{c}\text { Internal } \\
\text { probe }\end{array}$ & & \\
\hline \multirow{2}{*}{ HUES3 } & sgRNA-1 & 37 & 5 & 5 & 2 & 2 & 2 & $5 \%$ \\
\hline & sgRNA-2 & 36 & 0 & - & - & - & - & - \\
\hline
\end{tabular}

The gene targeting efficiency was calculated as the ratio of the correctly targeted clones to the total selection resistant clones.

Figure 1. CRISPR/Cas9 nickase induces precise gene targeting through homologous recombination in hESCs using a long DNA donor template. (A) The endogenous human SFTPC locus. The arrow indicates SFTPC gene. The stop codon (TAG) and the two targeting sites (\#1 and \#2) of guide RNA are indicated. The locations of upstream and downstream probes for Southern blotting and the sizes of WT Ndel (N) and Spel (S) restriction fragments are shown. (B) The long DNA donor template. The sizes and locations of homologous arms are indicated. The IRES-Puro-pA fragment and the F5 flanked selection cassette were inserted between the stop codon and the polyA signal sequence of SFTPC gene. Primers ( $a$ and $b$ ) used to screen homologous recombination at upstream arm are indicated. (C) The configuration of the knock-in allele. The location of internal probe for Southern blotting and the sizes of mutant $\mathrm{Ndel}$ and Spel restriction fragments are indicated. Primers (c, $d$ and e) used to screen clones after Flp transient expression are shown. (D) Southern blotting analysis of the clones with HR at upstream arm identified by PCR screening. Genomic DNA was digested with Ndel or Spel and hybridized to the upstream, internal and downstream probes sequentially with stripping between probes. The WT and KI bands are indicated. Bands generated by single-crossover at the upstream homologous arm are indicated by asterisks. (E) Excision of selection cassette was confirmed by PCR analysis. Flp expression plasmid was introduced into the HUES3 KI cells by electroporation. Genomic DNA isolated from individual clones was examined by PCR. Primers $c$ and $d$ amplify a 264-bp fragment in KI cells with the selection cassette, while primers $\mathrm{c}$ and e amplify a 329-bp fragment in $\mathrm{KI}$ cells after the selection cassette is excised. BAC plasmid without selection cassette was used as a positive control. (F) Summary of the frequency of homologous recombination in hESCs.

mediated gene targeting with wild type Cas9 that enables homozygous targeting of both alleles, no homozygous knock-in clones were identified in this study. To achieve homozygous knock-in clones with long DNA templates, a "double nicking" strategy might be employed with paired offset guide RNAs to significantly increase the homozygous targeting efficiency (Ran et al., 2013). We found three single- crossover clones with homologous recombination occurred only at the $5^{\prime}$ homologous arm. Therefore, our findings highlight the importance to confirm the homologous recombination events at both arms of the introduced donor DNA. In this context, in order to identify correctly targeted clones, both the upstream and downstream HR events as well as random integration of the template should be verified. 
In summary, our studies indicate that Cas9n can achieve precise gene targeting via HR in hESCs with a long DNA template, allowing high fidelity and complex genetic manipulation of hESCs. One such application is to develop lineage-specific reporter lines to trace the lineage differentiation of hESCs.

\section{FOOTNOTES}

We thank Dr. Zhao Chen for helpful discussion. This work was supported by Grants from National Natural Science Foundation of China (Grant Nos. 81172828 and 81372494) and from California Institute for Regenerative Medicine (TR3-05559).

Zhili Rong, Shengyun Zhu, Yang Xu and Xuemei Fu declare that they have no conflict of interest. All hESC work in this study has been approved by the Institutional Embryonic Stem Cell Research Oversight Committee (ESCRO) of University of California, San Diego and Shenzhen Children's Hospital.

Zhili Rong ${ }^{2}$, Shengyun Zhu ${ }^{1}$, Yang $\mathrm{Xu}^{2}$, Xuemei $\mathrm{Fu}^{1,3 凶}$

1 Shenzhen Children's Hospital, 7019 Yitian Road, Shenzhen 518026, China

2 Division of Biological Sciences, University of California, San Diego, 9500 Gilman Drive, La Jolla, CA 92093, USA

${ }^{3}$ Chongqing Medical University, Chongqing 400016, China

$\bowtie$ Correspondence: fxmzj2004@163.com (X. Fu)

\section{OPEN ACCESS}

This article is distributed under the terms of the Creative Commons Attribution License which permits any use, distribution, and reproduction in any medium, provided the original author(s) and the source are credited.

\section{REFERENCES}

Cong L, Ran FA, Cox D, Lin S, Barretto R et al (2013) Science 339:819-823

Degryse E, De Santi MM, Dietrich M, Hadji DA, Spetz JF et al (1996) Transgenic Res 5:139-143

Ding Q, Regan SN, Xia Y, Oostrom LA, Cowan CA et al (2013) Cell Stem Cell 12:393-394

Gaj T, Gersbach CA, Barbas CF 3rd (2013) Trends Biotechnol 31:397-405

Hockemeyer D, Soldner F, Beard C, Gao Q, Mitalipova M et al (2009) Nat Biotechnol 27:851-857

Hockemeyer D, Wang H, Kiani S, Lai CS, Gao Q et al (2011) Nat Biotechnol 29:731-734

Hsu PD, Scott DA, Weinstein JA, Ran FA, Konermann S et al (2013) Nat Biotechnol 31:827-832

Hwang WY, Fu Y, Reyon D, Maeder ML, Tsai SQ et al (2013) Nat Biotechnol 31:227-229

Jinek M, Chylinski K, Fonfara I, Hauer M, Doudna JA et al (2012) Science 337:816-821

Mali P, Aach J, Stranges PB, Esvelt KM, Moosburner M et al (2013a) Nat Biotechnol 31:833-838

Mali P, Yang L, Esvelt KM, Aach J, Guell M et al (2013b) Science 339:823-826

Niu Y, Shen B, Cui Y, Chen Y, Wang J et al (2014) Cell. doi:10.1016/j. cell.2014.01.027

Ran FA, Hsu PD, Lin CY, Gootenberg JS, Konermann S et al (2013) Cell 154:1380-1389

Song H, Chung SK, Xu Y (2010) Cell Stem Cell 6:80-89

Wang H, Yang H, Shivalila CS, Dawlaty MM, Cheng AW et al (2013) Cell 153:910-918

Electronic supplementary material The online version of this article (doi:10.1007/s13238-014-0032-5) contains supplementary material, which is available to authorized users. 